\title{
Trace level detection and quantification of crystalline silica in an amorphous silica matrix with ${ }^{29}$ Si NMR
}

\author{
Sambhu Radhakrishnan $\ddagger^{, a, b}$, Henri Colaux $\ddagger^{\mathrm{b}}$, C. Vinod Chandran $\ddagger^{\mathrm{a}, \mathrm{b}}$, Dirk Dom ${ }^{\mathrm{a}, \mathrm{b}}$, Loes Verheyden ${ }^{\mathrm{b}}$, \\ Francis Taulelle $^{\mathrm{b}}$, Johan Martens ${ }^{\mathrm{b}}$ and Eric Breynaert ${ }^{\mathrm{a}, \mathrm{b}}$ \\ ${ }^{a}$ NMRCoRe, Celestijnenlaan 200F, box 2461, 3001 Leuven, Belgium. \\ ${ }^{\mathrm{b}}$ Center for Surface Chemistry and Catalysis - Characterisation and Application Team (COK-kat), Department of Microbial \\ and Molecular Systems (M2S), KU Leuven, Celestijnenlaan 200F, box 2461, 3001 Leuven, Belgium.
}

Corresponding author E-mail: eric.breynaert@kuleuven.be

\begin{abstract}
A protocol for the detection by NMR spectroscopy of trace amounts of quartz in amorphous silica gels was developed and tested on commercially available samples. Using ${ }^{29} \mathrm{Si}$ MAS NMR spectroscopy with CPMG acquisition and standard addition of crystalline quartz, quantitative detection of quartz concentrations down to $0.1 \%$ wt. was achieved. CPMG permitted to suppress the amorphous silica derived signal, benefitting from the extremely long $T_{2}$ relaxation time of quartz in ${ }^{29} \mathrm{Si}$, and hence dramatically increasing the sensitivity. Dedicated post-processing exploiting the known CPMG spikelet frequencies allowed to probe the nearabsence of quartz in commercial, $100 \%$ silica samples, enabling to assess conformity of unknown samples to EU legislation (REACH).
\end{abstract}

Amorphous silica is commonly used in large scale applications: as desiccant, as filler in elastomers, for controlled release of drugs or as component in pest control systems. ${ }^{1-7}$ Quartz and other forms of respirable crystalline silica (RCS), on the contrary, are known to cause silicosis, lung cancer and chronic obstructive pulmonary disease (COPD) ${ }^{8-10}$ European Union regulations [REACH], require silica gel manufacturers to certify the absence of crystalline $\mathrm{SiO}_{2}$ in their products, defining a maximum allowed concentration of $0.1 \%$ wt. The most common method to assess crystalline material in an otherwise amorphous sample is X-ray diffraction. Methods used to determine the quartz /cristobalite content in dust, collected in workplace atmospheres, are Fourier transform infrared spectroscopy (FTIR) and X-ray diffraction (XRD) ${ }^{11,12}$ Reference standards for both methods are described in: AFNOR- XP X 43-243, AFNOR XP X 43-295, MDHS 101, NIOSH 7602 and NIOSH 7500.

With the maximum allowed concentration in amorphous silica (0.1\%wt.), XRD suffers from sensitivity. While in X-ray diffraction, one typically resorts to a higher photon flux source (e.g. synchrotron) to increase the signal to noise ratio beyond that of standard lab-instrument measurements, this paper reports on a new method using solid state nuclear magnetic resonance spectroscopy (ssNMR) under magic angle spinning to quantify the concentration of crystalline $\mathrm{SiO}_{2}$ in a $100 \%$ wt. $\mathrm{SiO}_{2}$ matrix down to a level of $0.1 \%$ wt.

Solid state NMR spectroscopy is largely used to characterize materials, especially to assess their chemical composition and chemical functionalities. It excels in the measurement of relative amounts of individual components in complex mixtures, the main reason being the independence of electromagnetic energy absorption by a given nucleus on the type of chemical compound it is part of. ${ }^{13}$ Proton $\left({ }^{1} \mathrm{H}\right)$ ssNMR is regularly used in food and pharmaceutical sciences to detect impurities and quantify their fraction relative to the main compound. ${ }^{14-16}$ Also absolute NMR quantification has been demonstrated, ${ }^{13,15-18}$ for example of the $\mathrm{H}_{2} \mathrm{O}$ content in microporous materials. ${ }^{19}$

However, quantitative detection of silicon remains a challenge because of the low sensitivity of the ${ }^{29} \mathrm{Si}$ nucleus, resulting from its low natural abundance $(4.7 \%)$ and low gyromagnetic ratio. Another important factor is its typically long longitudinal relaxation time $\left(T_{1}\right)$, requiring long repetition delays when using conventional direct NMR detection.

There are two types of relaxation mechanisms in NMR: the spin-lattice (also called longitudinal relaxation with a time constant $T_{1}$, i.e. relaxation along the direction of the static magnetic field) and spin-spin relaxation (also known as transverse relaxation with a time constant $T_{2}$, i.e. relaxation in the xy plane). For samples with long and similar $T_{1}$ for all components, (pre)-saturation can be used to obtain quantitative results without taking into account relaxation delays of $5 \times T_{1}$. Pre-saturation involves applying a series of $90^{\circ}$ pulses with very short delays in between, applied along the $\mathrm{x}$-axis, to induce a zero net magnetization on the z-axis. Subsequently, the magnetization is allowed to evolve over constant time before applying a final $\pi / 2$ pulse, yielding an identical net $\mathrm{z}$ - magnetization for every scan. In case components with highly different $T_{l}$ are present in the sample, internal quantitativity would however be lost upon (pre)-saturation. Absolute quantification is however still possible by standard addition of components with similar $T_{l}$ as the component of interest.

While long $T_{1}$ is typically troublesome, as it leads to long experiment times and low sensitivity per unit of time for classical direct NMR detection, in the present case, the extremely long $T_{1}$ of ${ }^{29} \mathrm{Si}$ contained in crystalline silicates can be turned into an advantage. $T_{1}$ values for quartz and other dense, crystalline pure 
a

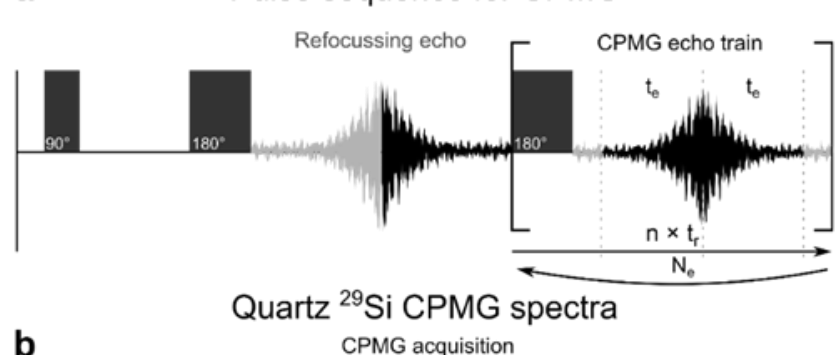

b

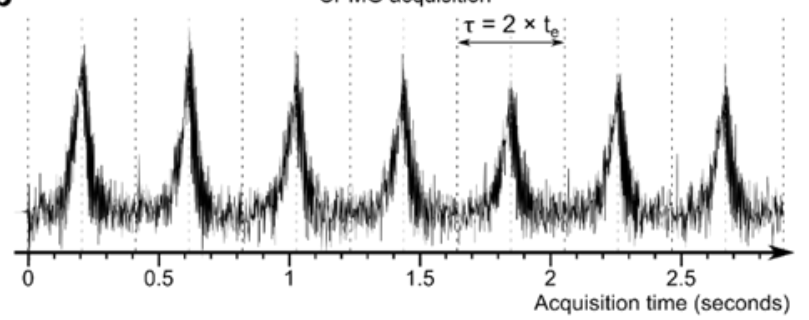

C
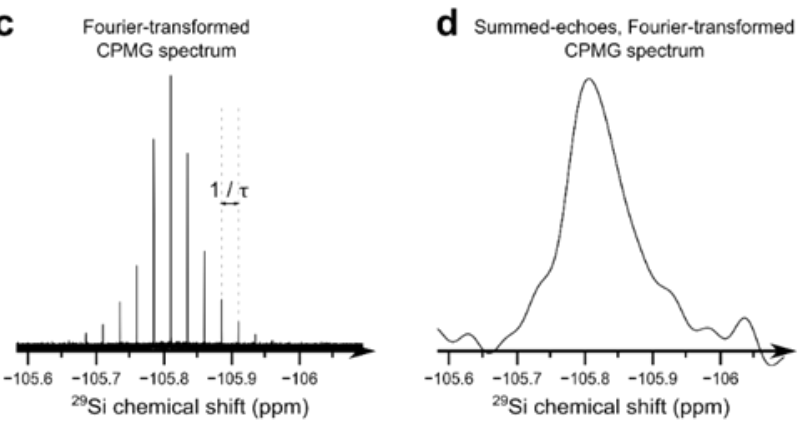

Figure 1. (a) Pulse sequence for CPMG detection. Values used in the experiments reported here for echo delay $\left(\tau=2 * t_{e}\right)$, number of echoes $\left(\mathrm{N}_{\mathrm{e}}\right)$, time of a rotor cycle ( $\left.\mathrm{t}_{\mathrm{r}}\right)$ and number of rotor cycles $(\mathrm{n})$ were $240 \mathrm{~ms}, 960,400 \mu \mathrm{s}$ and 600 respectively.; (b) quartz ${ }^{29} \mathrm{Si}$ CPMG echoes recorded; (c) Fourier transformed CPMG spectrum and (d) Summed-echoes, Fourier transformed spectrum.

silicate materials can be in the order of magnitude of hours, as compared to minutes for amorphous phases. Provided, the residual longitudinal magnetisation remains observable, a contrast in $T_{1}$ could be exploited as a filter to remove the signal of the amorphous phase.

While the slow $T_{1}$ relaxation (long $T_{1}$ ) offers options for signal filtering, in some cases, the associated long $T_{2}$ relaxation time constant can be exploited to enhance the sensitivity of the measurement. In cases where $T_{1}$ and $T_{2}$ are suitably long, solid state NMR sensitivity can be enhanced by exploiting multiple echo, multiple acquisition schemes like the Carr-PurcellMeiboom-Gill (CPMG) sequence. ${ }^{20,21}$ This acquisition scheme takes the advantage of the long $T_{2}$, to refocus the magnetization using a sequence of $180^{\circ}$ pulses at specific intervals (Figure 1). It allows to record the signal multiple times in a single scan, for acquisition times longer than the usual free induction decay (FID) observed following the direct-excitation measurement, thus increasing the total signal acquired per scan and in turn yielding a higher signal to noise ratio (SNR) per unit time. Fourier transformation of the train of CPMG echoes, leads to splitting of NMR pattern into a series of sharp peaks separated by a constant value $(1 / \tau)$, referred to as "spikelets" (see Figure 1c). This train of echoes can be used as it is, taking advantage of the narrow peak width, or can be summed and Fourier transformed to obtain the regular-looking NMR spectrum. The $\mathrm{S} / \mathrm{N}$ ratio of the spikelet spectrum however exceeds its summed regular- looking counterpart, because the integrated intensity of the classical spectrum is now concentrated into the spikelets. ${ }^{22}$ In addition, CPMG enables $T_{2}$ filtering, i.e., by choosing a long enough echo delay (time between subsequent $180^{\circ}$ pulses), components exhibiting a short $T_{2}$ can selectively be removed from the spectrum to obtain spectra containing only the component with the longest $T_{2}$, that is, the signal from the crystalline sample. ${ }^{23}$ In the present work, the long $T_{2}$ of quartz is exploited as a filter to selectively observe the quartz resonances.

Whereas the $T_{1}$ and $T_{2}$ relaxation properties of silicates offer options for signal filtering and sensitivity enhancement, they also impact absolute NMR quantification, in addition to the $Q$ factor, filling factor and probe calibration. ${ }^{19}$ In the present case, the solid state NMR based absolute quantification is also highly dependent on spin relaxation phenomena.

Crystalline silica phases (quartz, cristobalite and coesite) exclusively contain $\mathrm{Q}_{4} \mathrm{Si}$ species $\left(\mathrm{Si}-(\mathrm{O}-\mathrm{Si})_{4}\right)$, exhibiting chemical shifts between -100 and $-115 \mathrm{ppm}$. Quartz, for example, shows a single ${ }^{29}$ Si MAS NMR peak between -105.8 (this work) and $-107.1 \mathrm{ppm}$ with respect to TMS (tetra methyl silane), corresponding to tetrahedrally coordinated $\mathrm{Si}^{24,25}$ Cristobalite ${ }^{29} \mathrm{Si}$ resonates at around $-108.5 \mathrm{ppm} .{ }^{24}$ Coesite has two $\mathrm{Si}$ sites and shows two ${ }^{29} \mathrm{Si}$ resonances at -108.1 and $-113.9 \mathrm{ppm} .{ }^{24}$ Since there are not many crystalline pure silica references available, quartz makes a suitable standard system for a comparative investigation in the present issue. The $T_{1}$ of the ${ }^{29} \mathrm{Si}$ resonance of crystalline silica such as quartz usually is found to be of the order of magnitude of hours, slightly dependent on the external static magnetic field strengths, crystallinity, defect concentration and presence of impurities in the sample. In contrast, the $T_{1}$ relaxation constants for amorphous silicon compounds are found to be of the order of minutes instead of hours, hence enabling to discriminate between crystalline and amorphous silicon based on $T_{1}$, in addition to the apparent difference in line broadening, which also could result from a chemical shift distribution of crystalline components with nearly overlapping signals. ${ }^{26}$.

In the present study, pre-saturation was combined with CPMG acquisition to achieve selective, quantitative detection of crystalline silica in an amorphous $100 \%$ silica matrix at natural abundance.

\section{EXPERIMENTAL}

${ }^{29} \mathrm{Si}$ NMR spectra were acquired on a Bruker Avance III $500 \mathrm{MHz}$ spectrometer, equipped with $4 \mathrm{~mm} \mathrm{H} / \mathrm{X} / \mathrm{Y}$ probe and a $7 \mathrm{~mm} \mathrm{H} / \mathrm{X} / \mathrm{Y}$ probe, operating at ${ }^{29} \mathrm{Si}$ frequency of $99.5 \mathrm{MHz}$. The samples were filled in $\mathrm{ZrO}_{2}$ rotor $(4 \mathrm{~mm} / 7 \mathrm{~mm})$ and spun at $10 \mathrm{kHz}(4 \mathrm{~mm})$ or 2.5 $\mathrm{kHz}(7 \mathrm{~mm})$ and the rotor periods (tr) are $100 \mu \mathrm{s}$ and $400 \mu \mathrm{s}$, respectively. For $T_{1}$ determination of quartz, a $4 \mathrm{~mm}$ rotor was packed with ground crystalline alpha quartz and CPMG spectra were recorded with variable recycle delays $(8 \mathrm{~h}, 4 \mathrm{~h}, 2 \mathrm{~h} \& \mathrm{~h}$ ) after pre-saturation (100 pulses with $10 \mu \mathrm{s}$ intervals). The rotor was spun at $10 \mathrm{kHz}\left(\mathrm{t}_{\mathrm{r}}\right.$ $=100 \mu \mathrm{s}$ ), radiofrequency pulse of $52 \mathrm{kHz}$ was used and 8 transients were recorded. 32 echoes were acquired with an echo delay $(\tau)$ of $240 \mathrm{~ms}$. The integrated areas under the peaks were fitted with an exponential (Supporting information, Figure S1). ${ }^{29} \mathrm{Si}$ spectra were referenced to secondary reference, $\mathrm{Q} 8 \mathrm{M} 8$, which was referenced to primary reference, TMS $\left[\delta\left({ }^{29} \mathrm{Si}\right)=0 \mathrm{ppm}\right]$.

Standard addition samples were prepared via serial dilution. As a start, $10 \%$ wt. microcrystalline cellulose (KG-1000, Asahi Kasei) $90 \%$ wt. amorphous silica (SYLOID® 244 FP, Grace) was pre- 
pared via the 'equal parts method'. The cellulose was added to ensure the absence of any segregation during serial dilution or sample spinning and to facilitate compact rotor packing and hence stable magic angle spinning. Segregation might occur as result of the highly different densities of porous amorphous silica and quartz. Using the 10/90\%wt. cellulose/amorphous silica sample, quartz containing standard addition samples $(0.1 \%, 0.6 \%, 1 \%, 2 \%, 5 \%$ and $10 \%$ wt.) were prepared. Initially, the cellulose amorphous silica blend was mixed into crystalline alpha quartz (VWR) using the 'equal parts method', to reach a $10 \%$ wt. quartz sample. The quartz used was obtained by gently grinding alpha quartz (VWR) with a mortar and pestle and sieving the resulting powder through two meshes $(125$ and $250 \mu \mathrm{m})$ to selectively obtain particle sizes between 125 and $250 \mu \mathrm{m}$. Subsequently this sample was further diluted in the same way, to reach the other concentrations in the series $(5 \%, 2 \%, 1 \%, 0.6 \%$, and $0.1 \%$ wt. of Quartz).

The "equal parts method" used is a standard procedure in the pharmaceutical industry for creating homogeneous mixtures of solid components. In this method, equal volumes of two components are mixed using a mortar and a pestle. The blend is then "diluted" with again an equal volume of the most abundant component. This process is repeated until the desired final concentration is reached.

The quartz - silica mixtures were packed in a $7 \mathrm{~mm}$ rotor and spun at $2.5 \mathrm{kHz}\left(\mathrm{t}_{\mathrm{r}}=400 \mu \mathrm{s}\right)$. CPMG spectra were recorded with presaturation (100 pulses with $10 \mu$ s intervals). The variable delays between $180^{\circ}$ pulses ( $48 \mathrm{kHz} \mathrm{RF}$ pulse) in the CPMG experiments were optimized to selectively detect the signal of crystalline components (long $T_{2}$ ), filtering out the amorphous silica contributions. The experiments reported in this work used minimum 4 scans in combination with pre-saturation and 4 hours of recovery delay between scans, collecting 960 echoes $(\mathrm{Ne})$ per scan with an echo delay $(\tau)$ of $240 \mathrm{~ms}(\mathrm{n}=600$ rotor cycles). Number of transients rec-

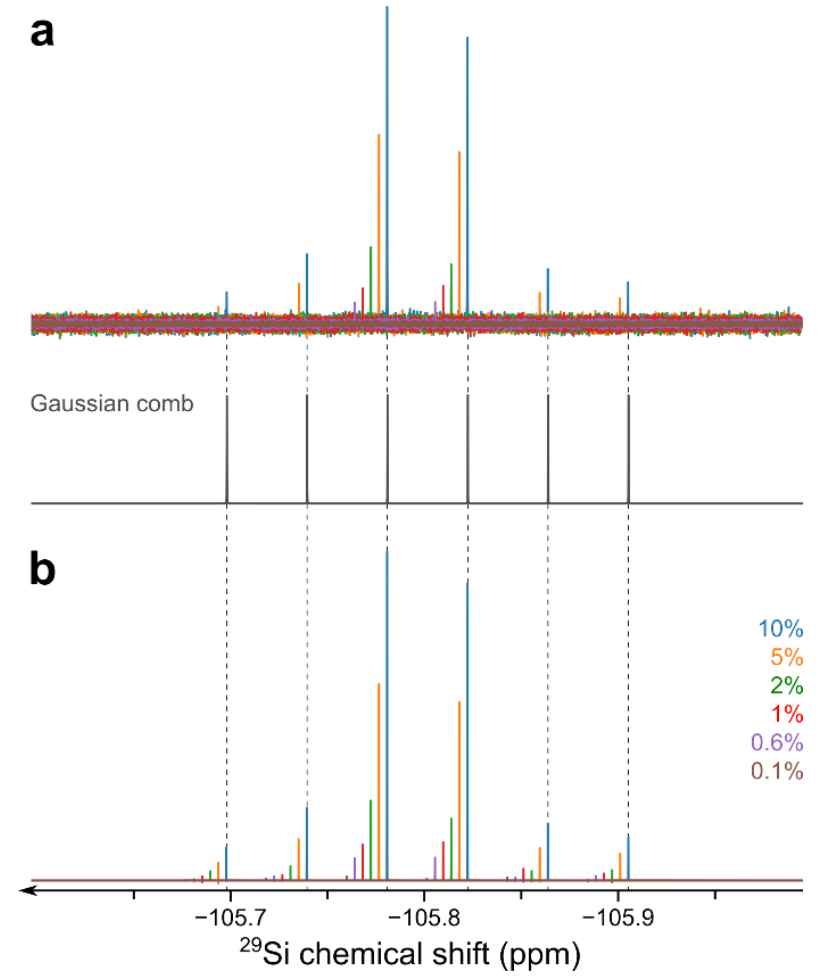

Figure 2. CPMG spectra centered around the quartz resonance (a) are the spectra before application of a Gaussian comb, and (b) are the combed spectra. For readability, the spectra were shifted by $-0.004,-0.008,-0.017,-0.021$, and $-0.025 \mathrm{ppm}$ for respectively the samples with standard additions of 5, 2, 1, 0.6 and $0.1 \%$ wt. of crystalline quartz.

orded were 4 for $10,5,2 \& 1 \%$ wt. quartz samples, while 12 and

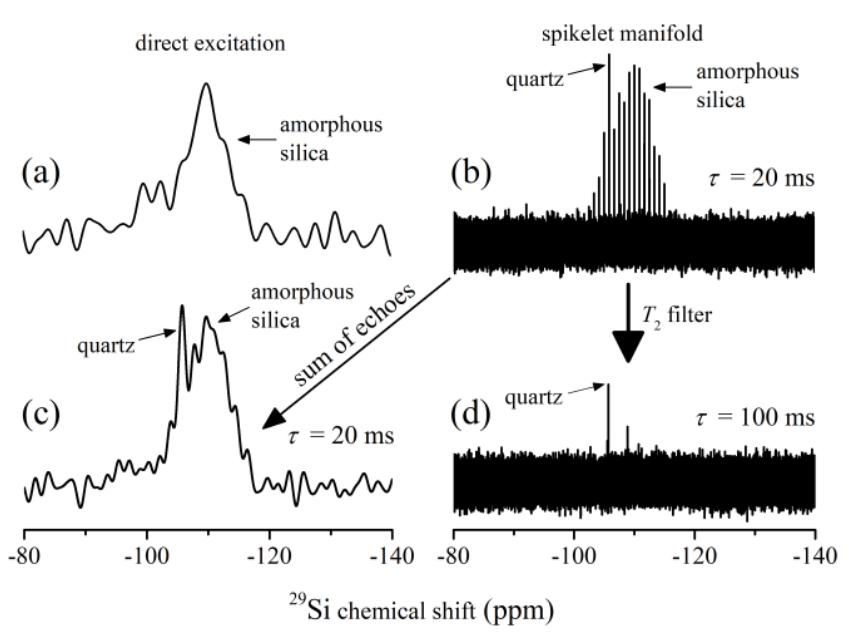

Figure $3 .{ }^{29} \mathrm{Si}$ NMR spectra of amorphous silica spiked with $5 \%$ of quartz collected with (a) single pulse direct excitation, (b) CPMG with an echo delay of $20 \mathrm{~ms}$ (c) and the sum of echoes and (d) CPMG with an echo delay of $100 \mathrm{~ms}$. Values used in the experiments reported here number of echoes $\left(\mathrm{N}_{\mathrm{e}}\right)$ and time of a rotor cycle (tr) were 100 and $100 \mu$ s respectively. Number of rotor cycles for the echo was $200(\tau=20 \mathrm{~ms})$ and $1000(\tau=100 \mathrm{~ms})$ for figure $3 \mathrm{a}-\mathrm{c}$ and figure $3 \mathrm{~d}$ respectively.

32 transients were acquired for the $0.6 \%$ wt. and $0.1 \%$ wt. mixtures respectively. The CPMG pulse sequence, echo train and Fourier transformed spectrum of pure quartz recorded with CPMG are depicted in Figure 1.

The drift of the static magnetic field was measured on ${ }^{1} \mathrm{H}$ spectra that were acquired between every 4 scans (16h) and average drift was estimated. The drift was compensated by multiplying the FID by a complex exponential at a frequency opposite to the drift using a python script.

CPMG detection concentrates all signal area contained in a classical NMR spectra into spikelets. In CPMG-detected spectra, separation of the spikelets associated with a peak in the classical NMR spectrum is determined by the acquisition parameters. As for a specific NMR signal (e.g. derived from quartz), the narrow spectral ranges where signal intensity can occur are fully determined by the acquisition parameters. Hence, it is possible to significantly reduce the error in the determination of the integrated intensity by a point-by-point multiplication of all processed spectra (after corrections for the phase and for the drift of the static magnetic field) by a comb of Gaussian functions centred on the chemical shift range of quartz. This comb consists of a series of Gaussian line shapes regularly spaced with identical widths and intensity, as shown in Figure 2 (Gaussian line shapes shifted by respectively $-0.1,-0.06,-0.02$, $+0.02,+0.06$ and $0.1 \mathrm{ppm}$ from $-105.8 \mathrm{ppm}$, chemical shift of the centre of the CPMG spikelet spectrum of Quartz). ${ }^{27-29}$ The amplitude and width of all Gaussians were determined from the most intense peak of the spectrum (thus with the highest standard addition), and applied on all other visible peaks in the spectrum. The Gaussian comb established this way was subsequently used on all other spectra. The integrated intensity was obtained by integrating the complex spikelet spectrum after 


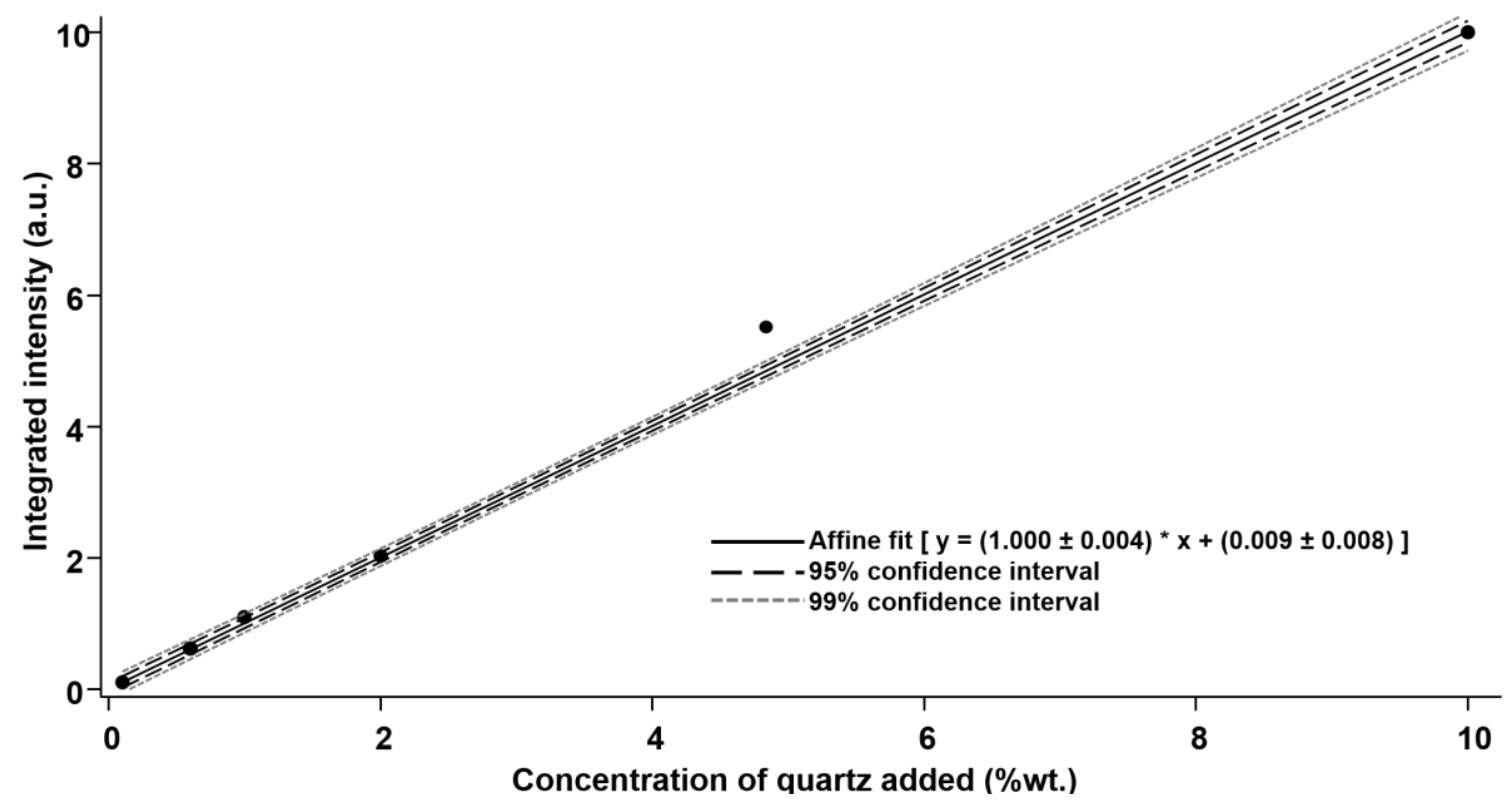

Figure 4. Plot of the scaled integrated intensity for the different quartz - silica - cellulose mixtures given in Table 1, against the added quartz concentration, with the corresponding affine fit and the $95 \%$ and $99 \%$ confidence intervals. Linear fitting was performed without the $5 \%$ wt. data point and with error minimization iterations taking into account the noise levels on the intensities.

multiplication by the Gaussian comb. In standard addition samples where the signal was difficult to discern in the noise, the zero order phase was optimised to maximise the integrated amplitude (e.g. $0.1 \%$ wt.). ${ }^{30-32}$ Integrated areas (a.u) for NMR quantification were normalized to 10 , accounting for the different number of scans, and plotted against \%wt. of crystalline quartz added. The regression was performed with error minimization iterations in Wavemetrics Igor Pro software, taking into account the uncertainty on the intensities obtained by sampling the noise in the spectrum with the Gaussian comb. The total crystalline silica content was subsequently determined using the slope and intercept from the linear regression.

\section{RESULTS AND DISCUSSION}

${ }^{29} \mathrm{Si} T_{1}$ of quartz was determined using a series of experiments with varying initial delays (vide supra). This curve is shown in Figure S1. As expected, this resulted in an exponential increase of the signal intensity (and area) with increasing recovery delay. A mono-exponential growth function employed for fitting the integrated area under the peaks yielded a $T_{1}$ relaxation time of 9.3 hours. Experiments were performed with different CPMG echo delays to filter out the broad amorphous silica resonances and retain only sharp crystalline silica resonances (Figure 3). The direct excitation ${ }^{29} \mathrm{Si}$ NMR spectrum of $5 \%$ wt. quartz added to amorphous silica is depicted in Figure 3a, showing the broad silica resonances, while the sharp quartz resonance is not immediately visible. Figure $3 \mathrm{~b}$ and Figure $3 \mathrm{c}$ respectively show the CPMG spikelet pattern recorded with an echo delay of 20 $\mathrm{ms}$ and the Fourier transformation of summed echoes. The resonances of the amorphous silica as well as the spiked crystalline quartz are clearly recognizable in both the spectra. Comparing Figure $3 c$ to Figure $3 a$, the signal of the amorphous silica is suppressed, while the the resonance of quartz is enhanced because its long $T_{2}$ enables accumulation of higher number of echoes and hence a higher integrated signal intensity per scan. Figure $3 \mathrm{~d}$ shows the CPMG spikelet pattern recorded with a longer echo delay (100 ms). The $T_{2}$ filtering effect of CPMG is evident, as resonances of the short $T_{2}$ component (amorphous silica) is not present, allowing the selective acquisition of the long $T_{2}$ component alone. Performing the CPMG detected acquisition, all signal area of the quartz peak indicated in Figure $3 \mathrm{c}$ is transformed into the pattern of spikelets with an inter-spikelet spacing determined by the acquisition parameters (Figure $2 b$ and Figure 3d).

In the ${ }^{29} \mathrm{Si} \mathrm{CPMG}$ experiments reported here, the standard recovery delay of $5 \times T_{1}$ used in experiments was not taken into account, as this would have extended the total experiment time immensely. Instead pre-saturation was applied, allowing to reproducibly use shorter relaxation delays and acquire more echoes per unit of time, finally enhancing the signal intensity per unit of time.

Figure 2 shows ${ }^{29} \mathrm{Si}$ CPMG spectra of amorphous silica SYLOID® 244 FP with 10.0, 5.0, 2.0, 1.0, 0.6 and $0.1 \%$ wt. of crystalline quartz added via standard addition. The purpose of these experiments is two-fold:

- Verify that the signal-to-noise ratio varies linearly with the proportion of $\alpha$-quartz added.

- Quantifying the detection limit of this experiment for this material and for a given acquisition time, that expresses the minimum fraction required for statistically relevant detection.

In order to estimate the uncertainty of each of the integrated intensities, the Gaussian comb previously defined was applied in spectral regions where no ${ }^{29} \mathrm{Si}$ signal arising from crystalline species with long $T_{2}$ can be present and consequently only noise is sampled (Gaussian comb was applied at 4 positions in the spectrum shifted by $-2.1,-0.8,0.8,2.1 \mathrm{ppm}$ respectively from the quartz peak centre at $-105.8 \mathrm{ppm}$ ). All complex points were summed and this value was taken as a sampling of the uncertainty on the absolute value of the signal obtained for the quartz in that sample. For every sample, the uncertainty measurement 
was performed on 4 distinct spectral regions (supra) and averaged. These averaged values were then used as uncertainties in the affine linear regression (Table 1).

Figures 4, S5 \& S6 and Table $1 \&$ S1 show the results of affine regressions of the crystalline silica concentration (\%wt.) as determined with ${ }^{29} \mathrm{Si}$ NMR versus the concentration of crystalline quartz (\%wt.) added via standard addition, for all samples based on SYLOID ${ }^{\circledR} 244$ FP. The regression evidences the linear correlation between the measured crystalline silica concentration and the concentration of crystalline quartz dosed into the samples via standard addition. As it can be observed in Figure 4 , the $5 \%$ wt. data point is the only point that does not fall into the $95 \%$ or the $99 \%$ confidence region of the 5-point regression, excluding this point. As there are no obvious arguments from the NMR acquisition, this discrepancy was assigned to an inaccuracy not related to the NMR acquisition, but to an experimental error in sample preparation. Affine regression was performed without including the $5 \%$ wt. data point and the results are depicted in Figure 4 and Table 1. The intercept $b$ of the affine regression $(y=a * x+b$; with $a=1.000 \pm 0.004$, $\mathrm{b}=0.009 \pm 0.008$ ), the uncertainty corresponding to one standard deviation) immediately demonstrate that the crystalline silica concentration of SYLOID $® 244 \mathrm{FP}$ is far below the limit of $0.1 \%$ wt.

Since the affine regression shown in Figure 4 yields an intercept $(b-$ value $)$ extremely near 0 , also a linear regression $(y=a * x)$ can be performed on the normalized dataset reported in Table 1. This linear regression results in a value of $\mathrm{a}=1.002 \pm 0.004$, again indicating that the amorphous silica sample tested does not contain a concentration of crystalline silica significantly different from $0 \%$ wt.

Table 1: ${ }^{29} \mathrm{Si}$ NMR determined crystalline silica concentration (\%wt.) versus the concentration of crystalline quartz (\%wt.) added via standard addition.

\begin{tabular}{|c|c|c|c|c|}
\hline $\begin{array}{c}\text { Conc. of } \\
\text { quartz } \\
\text { added } \\
\text { (\%wt.) }\end{array}$ & $\begin{array}{l}\text { Number } \\
\text { of scans }\end{array}$ & $\begin{array}{c}\text { Integrated } \\
\text { intensity } \\
\text { (a.u.) }\end{array}$ & $\begin{array}{c}\text { Noise } \\
\text { level } \\
\text { (a.u.) }^{\mathrm{a}}\end{array}$ & $\begin{array}{l}\text { Conc. of } \\
\text { quartz } \\
\text { measured } \\
(\% \text { wt. })^{b}\end{array}$ \\
\hline 0.1 & 32 & 0.104 & 0.009 & 0.093 \\
\hline 0.6 & 12 & 0.621 & 0.019 & 0.606 \\
\hline 1 & 4 & 1.094 & 0.062 & 1.076 \\
\hline 2 & 4 & 2.026 & 0.033 & 2.002 \\
\hline 5 & 4 & 5.527 & 0.078 & 5.478 \\
\hline 10 & 4 & 10 & 0.044 & 9.919 \\
\hline
\end{tabular}

${ }^{a}$ error estimated by Gaussian comb multiplication at regions without ${ }^{29} \mathrm{Si}$ signal.

${ }^{\mathrm{b}}$ concentration calculated using the slope and intercept reported in figure 4 .

\section{CONCLUSIONS}

Solid state NMR methodology was developed to quantitatively determine the trace amount concentrations of crystalline silica in a mixture with amorphous silica. $T_{2}$-filtered ${ }^{29} \mathrm{Si}$ NMR using CPMG acquisition scheme allowed separation of the sharp resonance of crystalline silica from the broad resonances of amorphous silica. A post-processing procedure using a Gaussian comb functions was used to extract the resonances of crystalline component in mixtures with its concentration as low as $0.1 \%$ wt. The affine regression of a standard addition series $(0.1,0.6,1,2,5$ and $10 \%$ wt.) using finely ground crystalline quartz as reference for crystalline silica demonstrated that the content of crystalline silica in the commercial amorphous silica is significantly below the limit of $0.1 \%$ wt.

\section{ASSOCIATED CONTENT}

\section{Supporting Information}

CPMG spikelet summation procedure, longitudinal relaxation of ${ }^{29} \mathrm{Si}$ of quartz at a static magnetic field of $11.7 \mathrm{~T}$, drift corrected and Gaussian comb multiplied CPMG spectra of different quartz amorphous silica mixtures. The Supporting Information is available free of charge at charge via the Internet at http://pubs.acs.org.

\section{AUTHOR INFORMATION}

Corresponding Author

* Email: eric.breynaert@kuleuven.be

Orcid

Henri Colaux: 0000-0002-4794-0572

Sambhu Radhakrishnan: 0000-0002-0274-2759

C. Vinod Chandran: 0000-0003-4074-8833

Johan A. Martens: 0000-0002-9292-2357

Francis Taulelle: 0000-0002-8263-4802

Eric Breynaert: 0000-0003-3499-0455

\section{Author Contributions}

$\$ \mathrm{HC}, \mathrm{SR}$ and CVC contributed equally to the manuscript.

The manuscript was written through contributions of all authors. All authors have given approval to the final version of the manuscript.

\section{ACKNOWLEDGMENT}

This work was supported by the Hercules Foundation (AKUL/13/21), and by the Flemish Government, department EWI via the Hermes Fund (AH.2016.134). J. A. M. acknowledge the Flemish Government for long-term structural funding (Methusalem).

\section{REFERENCES}

(1) Barik, T. K.; Sahu, B.; Swain, V. Nanosilica - From Medicine to Pest Control. Parasitology Research. Springer July 29, 2008, pp 253-258. https://doi.org/10.1007/s00436-008-0975-7.

Shawir, M.; le Patourel, G. N. J.; Moustafa, F. I. Amorphous Silica as an Additive to Dust Formulations of Insecticides for Stored Grain Pest Control. J. Stored Prod. Res. 1988, 24 (3), 123130. https://doi.org/10.1016/0022-474X(88)90009-4.

Verraedt, E.; van den Mooter, G.; Martens, J. A. Novel Amorphous Microporous Silica Spheres for Controlled Release Applications. J. Pharm. Sci. 2011, 100 (10), 4295-4301. https://doi.org/10.1002/jps.22617.

Verraedt, E.; Braem, A.; Chaudhari, A.; Thevissen, K.; Adams, E.; Van Mellaert, L.; Cammue, B. P. A.; Duyck, J.; Anné, J.; Vleugels, J.; et al. Controlled Release of Chlorhexidine Antiseptic from Microporous Amorphous Silica Applied in Open Porosity of an Implant Surface. Int. J. Pharm. 2011, 419 (1-2), 28-32. https://doi.org/10.1016/j.ijpharm.2011.06.053.

(5) León D., P.; Luis F., G.; Betty L., L.; Hess, M. Reinforcing of Elastomers with Mesoporous Silica. Macromol. Symp. 2006, 245-246 (1), 628-640. https://doi.org/10.1002/masy.200651390.

(6) Komarneni Sridhar; Prakash B. Malla. Silicagels of Controlled Pore Size as Desiccant Materials and Processes of Producing Same. US5364455A, September 22, 1992.

(7) Barua, S.; Gogoi, S.; Khan, R.; Karak, N. Silicon-Based 
Nanomaterials and Their Polymer Nanocomposites. In Nanomaterials and Polymer Nanocomposites: Raw Materials to Applications; $\quad$ Elsevier, 2018; pp 261-305. https://doi.org/10.1016/B978-0-12-814615-6.00008-4.

(8) Safe Silica | Crystalline Silica Information | Silicosis Prevention https://safesilica.eu/ (accessed Apr 21, 2020).

(9) Safety and Health Topics | Respirable Crystalline Silica Occupational Safety and Health Administration https://www.osha.gov/dsg/topics/silicacrystalline/ (accessed Apr 23, 2020).

(10) Merget, R.; Bauer, T.; Küpper, H.; Philippou, S.; Bauer, H.; Breitstadt, R.; Bruening, T. Health Hazards Due to the Inhalation of Amorphous Silica. Archives of Toxicology. Springer Verlag November 29, 2002, pp 625-634. https://doi.org/10.1007/s002040100266.

(11) Hart, J. F.; Autenrieth, D. A.; Cauda, E.; Chubb, L.; Spear, T. M.; Wock, S.; Rosenthal, S. A Comparison of Respirable Crystalline Silica Concentration Measurements Using a Direct-on-Filter Fourier Transform Infrared (FT-IR) Transmission Method vs. a Traditional Laboratory X-Ray Diffraction Method. J. Occup. Environ. Hyg. 2018, $15 \quad$ (10), 743-754. https://doi.org/10.1080/15459624.2018.1495334.

(12) Verma, D. K.; Johnson, D. M.; Tombe, K. Des. A Method for Determining Crystalline Silica in Bulk Samples by Fourier Transform Infrared Spectrophotometry. Ann. occup. Hyg 2002, 46 (7), 609-615. https://doi.org/10.1093/annhyg/mef077.

(13) Shoolery, J. N. Quantitative Measurements. In Encyclopedia of Magnetic Resonance; John Wiley \& Sons, Ltd: Chichester, UK, 2007. https://doi.org/10.1002/9780470034590.emrstm0436.

(14) Pauli, G. F.; Jaki, B. U.; Lankin, D. C. Quantitative 1H NMR: Development and Potential of a Method for Natural Products Analysis. J. Nat. Prod. 2005, 68 (1), 133-149. https://doi.org/10.1021/np0497301.

(15) Pauli, G. F.; Gödecke, T.; Jaki, B. U.; Lankin, D. C. Quantitative 1H NMR. Development and Potential of an Analytical Method: An Update. J. Nat. Prod. 2012, 75 (4), 834-851. https://doi.org/10.1021/np200993k.

(16) Pauli, G. F.; Chen, S.-N.; Simmler, C.; Lankin, D. C.; Gödecke, T.; Jaki, B. U.; Friesen, J. B.; McAlpine, J. B.; Napolitano, J. G. Importance of Purity Evaluation and the Potential of Quantitative 1H NMR as a Purity Assay. J. Med. Chem. 2014, 57 (22), 92209231. https://doi.org/10.1021/jm500734a.

(17) Evilia, R. F. Quantitative NMR Spectroscopy. Analytical Letters. Taylor \& Francis Group 2001, pp 2227-2236. https://doi.org/10.1081/AL-100107290.

(18) Singh, S.; Roy, R. The Application of Absolute Quantitative 1H NMR Spectroscopy in Drug Discovery and Development. Expert Opinion on Drug Discovery. Taylor and Francis Ltd July 2, 2016 pp 695-706. https://doi.org/10.1080/17460441.2016.1189899.

(19) Houlleberghs, M.; Hoffmann, A.; Dom, D.; Kirschhock, C. E. A.; Taulelle, F.; Martens, J. A.; Breynaert, E. Absolute Quantification of Water in Microporous Solids with $1 \mathrm{H}$ Magic Angle Spinning NMR and Standard Addition. Anal. Chem. 2017, 89 (13), 6940
6943. https://doi.org/10.1021/acs.analchem.7b01653.

(20) Carr, H. Y.; Purcell, E. M. Effects of Diffusion on Free Precession in Nuclear Magnetic Resonance Experiments. Phys. Rev. 1954, 94 (3), 630-638. https://doi.org/10.1103/PhysRev.94.630.

(21) Meiboom, S.; Gill, D. Modified Spin-Echo Method for Measuring Nuclear Relaxation Times. Rev. Sci. Instrum. 1958, 29 (8), 688-691. https://doi.org/10.1063/1.1716296.

(22) Siegel, R.; Nakashima, T. T.; Wasylishen, R. E. Signal-to-Noise Enhancement of NMR Spectra of Solids Using Multiple-Pulse Spin-Echo Experiments. Concepts Magn. Reson. Part A $\mathbf{2 0 0 5}$ 26A (2), 62-77. https://doi.org/10.1002/cmr.a.20038.

(23) Rastrelli, F.; Jha, S.; Mancin, F. Seeing through Macromolecules: T2-Filtered NMR for the Purity Assay of Functionalized Nanosystems and the Screening of Biofluids. J. Am. Chem. Soc. 2009, 131 (40), 14222-14224. https://doi.org/10.1021/ja904737r. Smith, J. V.; Blackwell, C. S. Nuclear Magnetic Resonance of Silica Polymorphs. Nature 1983, 303 (5914), 223-225. https://doi.org/10.1038/303223a0.

(25) Lippmaa, E.; Magi, M.; Samoson, A.; Engelhard, G.; Grimmer, A. R. Structural Studies of Silicates by Solid-State HighResolution 29Si NMR. J. Am. Chem. Soc. 1980, 102 (15), 48894893. https://doi.org/10.1021/ja00535a008.

(26) Léonardelli, S.; Facchini, L.; Fretigny, C.; Tougne, P.; Legrand, A. P. Silicon-29 Nuclear Magnetic Resonance Study of Silica. J. Am. Chem. Soc. 1992, 114 (16), 6412-6418. https://doi.org/10.1021/ja00042a018.

(27) Bessin, F.; Perego, A. M.; Staliunas, K.; Turitsyn, S. K.; Kudlinski, A.; Conforti, M.; Mussot, A. Gain-through-Filtering Enables Tuneable Frequency Comb Generation in Passive Optical Resonators. Nat. Commun. 2019, 10 (1), 1-6. https://doi.org/10.1038/s41467-019-12375-3.

(28) Murphy, M. T.; Locke, C. R.; Light, P. S.; Luiten, A. N.; Lawrence, J. S. Laser Frequency Comb Techniques for Precise Astronomical Spectroscopy. Mon. Not. R. Astron. Soc. 2012, 422 (1), 761-771. https://doi.org/10.1111/j.1365-2966.2012.20656.x.

(29) Yi, X.; Vahala, K.; Li, J.; Diddams, S.; Ycas, G.; Plavchan, P.; Leifer, S.; Sandhu, J.; Vasisht, G.; Chen, P.; et al. Demonstration of a Near-IR Line-Referenced Electro-Optical Laser Frequency Comb for Precision Radial Velocity Measurements in Astronomy. Nat. Commun. 2016, 7 (1), 1-9. https://doi.org/10.1038/ncomms10436.

(30) Marion, D. Timing and Related Artifacts in Multidimensional NMR. Concepts Magn. Reson. Part A 2012, $40 A$ (6), 326-340. https://doi.org/10.1002/cmr.a.21250.

(31) Binczyk, F.; Tarnawski, R.; Polanska, J. Strategies for Optimizing the Phase Correction Algorithms in Nuclear Magnetic Resonance Spectroscopy. Biomed. Eng. Online 2015 , 14 (2), S5. https://doi.org/10.1186/1475-925X-14-S2-S5.

(32) Chen, L.; Weng, Z.; Goh, L. Y.; Garland, M. An Efficient Algorithm for Automatic Phase Correction of NMR Spectra Based on Entropy Minimization. J. Magn. Reson. 2002, 158 (12), 164-168. https://doi.org/10.1016/S1090-7807(02)00069-1. 


\section{Graphical abstract:}
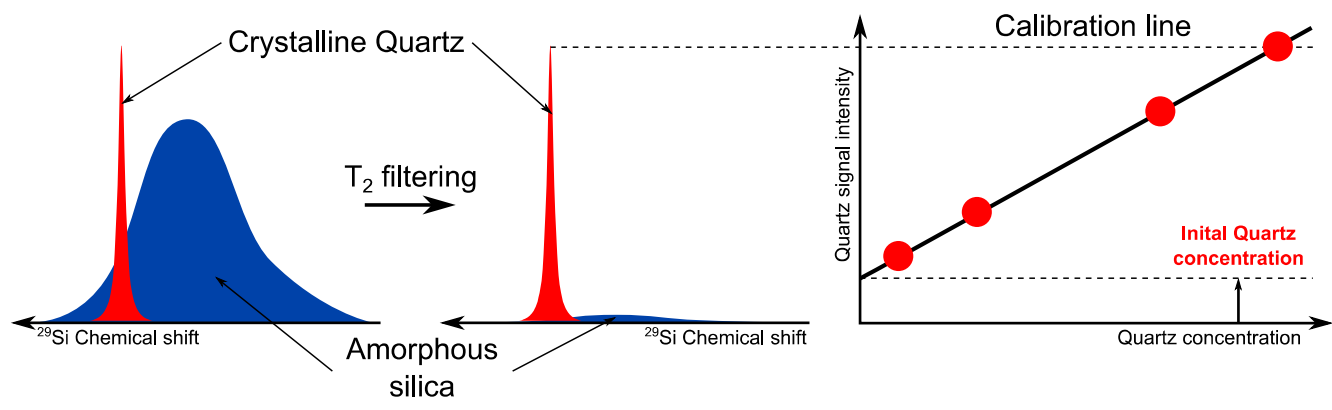

silica 